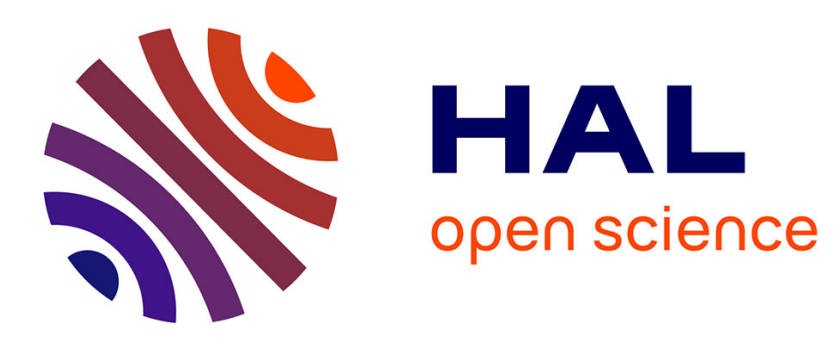

\title{
INTERACTION OF FAST HYDROGEN IONIC CLUSTERS WITH MATTER
}

M. Chevallier, N.V. de Castro Faria, B. Farizon-Mazuy, M. Gaillard, J.-C. Poizat, J. Remillieux

\section{- To cite this version:}

M. Chevallier, N.V. de Castro Faria, B. Farizon-Mazuy, M. Gaillard, J.-C. Poizat, et al.. INTERACTION OF FAST HYDROGEN IONIC CLUSTERS WITH MATTER. International Workshop On Mev And Kev Ions And Cluster Interactions With Surfaces And Materials 2, 1988, Orsay, France. pp.C2-189-C2-193, 10.1051/jphyscol:1989232 . jpa-00229430

\section{HAL Id: jpa-00229430 https://hal.science/jpa-00229430}

Submitted on 1 Jan 1989

HAL is a multi-disciplinary open access archive for the deposit and dissemination of scientific research documents, whether they are published or not. The documents may come from teaching and research institutions in France or abroad, or from public or private research centers.
L'archive ouverte pluridisciplinaire $\mathbf{H A L}$, est destinée au dépôt et à la diffusion de documents scientifiques de niveau recherche, publiés ou non, émanant des établissements d'enseignement et de recherche français ou étrangers, des laboratoires publics ou privés. 


\title{
INTERACTION OF FAST HYDROGEN IONIC CLUSTERS WITH MATTER
}

\author{
M. CHEVALLIER, N.V. de CASTRO FARIA, B. FARIZON-MAZUY, M.J. GAILLARD, \\ J.C. POIZAT and J. REMILLIEUX \\ Institut de Physique Nucléaire (and IN2P3), Université Claude Bernard \\ Lyon-1, 43, Bd du 11 Novembre 1918, F-69622 Villeurbanne Cedex, France
}

\begin{abstract}
Résumé - Les agrégats rapides d'hydrogène $\mathrm{H}^{+} \mathrm{n}^{\prime}$ interagissent pas avec la matière de la même manière que des protons de même vitesse. Nous présentons les résultats obtenus à Lyon concernant l'interaction d'agrégats légers d'hydrogène $(5 \leq \mathrm{n} \leq 23)$ avec une cible gazeuse ou une feuille mince, à des vitesses voisines de la vitesse de Bohr (dynamique de la fragmentation, état de charge des fragments émergents, section efficace de dissociation dans un gaz). Nous discutons aussi d'expériences futures et en particulier à plus haute énergie.

Abstract - Fast ionic clusters $\mathrm{H}^{+}$interact with matter in a specific way which is observed to deviate strongly from the interaction of atomic ions at the same velocity. We present some results obtained at lyon about foil and gas interactions of hydrogen clusters $(5<\mathrm{n}<23)$ at projectile velocities close to the Bohr velocity, i.e. dynamics of the cluster fragmentation, charge state of atomic fragments and absolute dissociation cross sections in gas. We also discuss future experiments specially at higher velocities.
\end{abstract}

Recent advances in experimental techniques allow to produce atom clusters of various sizes and compositions. This has created a new area of research since clusters can be regarded as large molecules or as fragments of condensed matter. The acceleration of clusters started when Henkes /1/ sent $\mathrm{CO}_{2}$ cluster ions through a potential difference up to $2 \mathrm{KV}$ and analysed their mass spectrum. These experiments soon led to various efforts to accelerate hydrogen ions for use in nuclear fusion technology. The hydrogen clusters are the simplest ionic molecular complexes. Their structure has generally been described as $\mathrm{H}_{2}$ molecules surrounding a $\mathrm{H}_{3}^{+}$ionic core, i.e. $\mathrm{H}_{3}^{+}\left(\mathrm{H}_{2}\right)_{n} / 2 /$.

Hydrogen cluster beams of energies ranging from 40 to $700 \mathrm{keV}$ are currently delivered by the Cockroft-Walton cluster accelerator of the Institut de Physique Nucléaire de Lyon /3/. The accelerated ionic cluster beam is selected in energy and mass by electrostatic and magnetic analysers.

In a first experiment $14 /$, we have measured the transmission of light hydrogen cluster ions through a argon gas target, for projectile velocities between 1.5 to $510^{8} \mathrm{~cm} / \mathrm{s}$, i.e. around the Bohr velocity. These measurements allowed us to study the dissociation cross-sections $\sigma_{d}$ as a function of the mass of the cluster and its velocity. Determining $\sigma_{d}$ is useful not only for practical reasons, but also because the velocity dependence of $\sigma_{d}$ for a given cluster can help understand the break up processes. Moreover the variation of $\sigma_{d}$ with $n$, the cluster proton number, at a given velocity, yields information on the cluster structure.

On figure 1, we show the mass dependence of the dissociation cross section for $20 \mathrm{keV} / \mathrm{p} \mathrm{H}_{\mathrm{n}}^{+}$clusters in an argon gas target. Note that this figure includes $\sigma_{d}$ for $12 \mathrm{keV} / \mathrm{p}$ clusters with $\mathrm{n}=21$ et 23 (square symbols) since we know that in this velocity range $\sigma_{d}$ remains almost independent of the velocity. If one excepts the $\sigma_{d}$ values obtained for $n=15$ and $n=19$, the variation of $\sigma_{d}$ with $n$ is very well described by the law $\sigma_{d}=K N^{\alpha}$ with $\alpha=0.66 \sim 2 / 3$ and $K=8.65 \AA^{2}$ (see figure 1 ). Since the cluster orientations with respect to the beam direction are randomly distributed, we may 


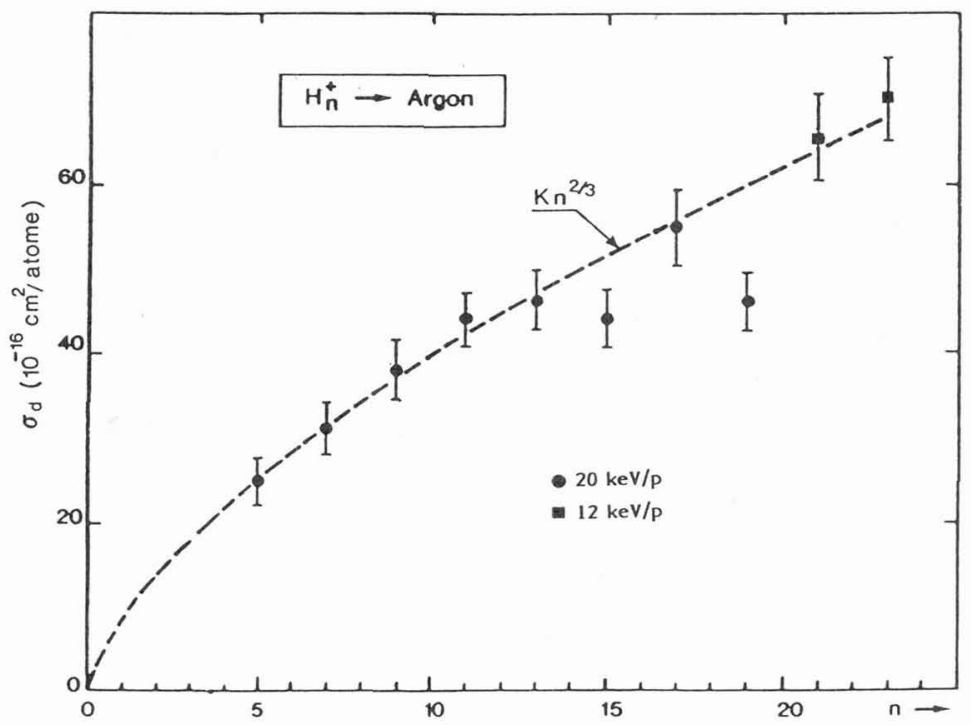

Figure 1 - Mass dependence of the dissociation cross section of $\mathrm{H}_{n}^{+}$clusters in argon gas. The dashed line corresponds to a fit with $a n^{2 / 3}$ law (see text).

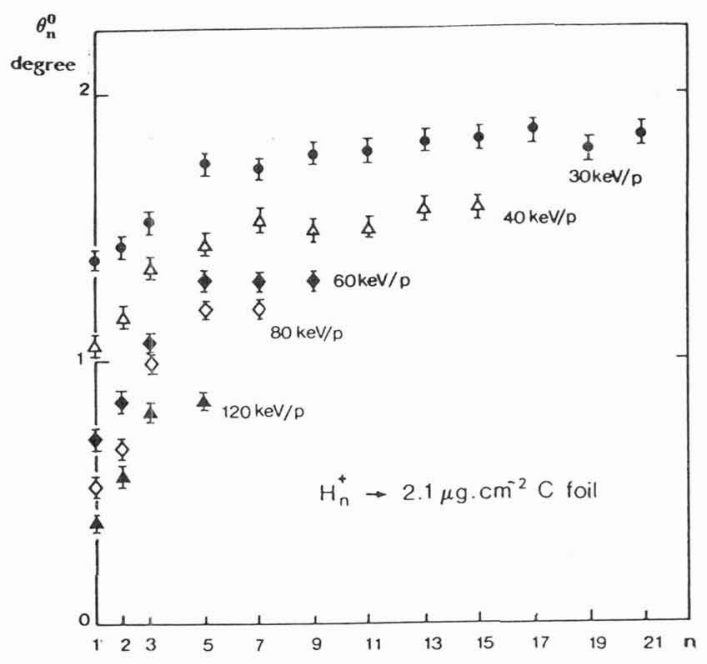

Figure 2 - Mass dependence of the FWHM of the angular distributions of neutral atoms resulting from the dissociation of 30 to 120 $\mathrm{keV} / \mathrm{p} \mathrm{H}_{n}^{+}$clusters in a $2.1 \mathrm{\mu g} \cdot \mathrm{cm}^{-2}$ thick carbon foil.

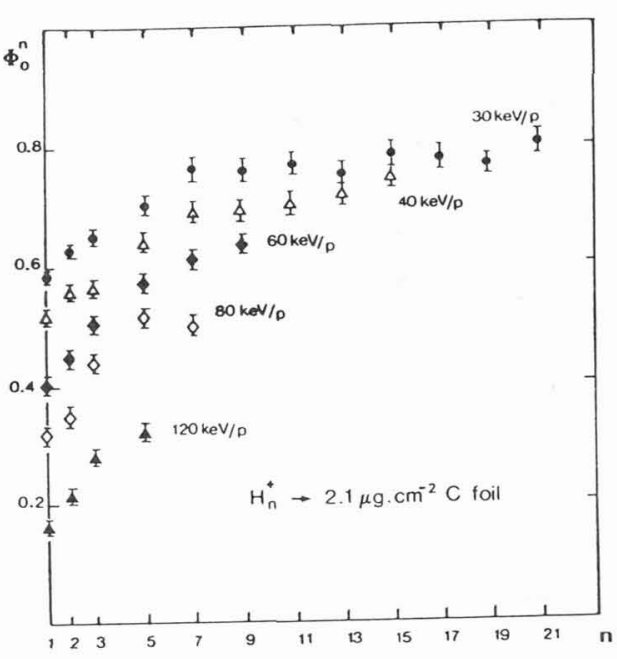

Figure 3 - Mass dependence of the neutral fraction in the atomic fragments resulting from the dissociation of 30 to $120 \mathrm{keV} / \mathrm{p} \mathrm{H}_{n}^{+}$ clusters in a $2.1 \mu \mathrm{g} . \mathrm{cm}^{-2}$ thick carbon foil. 
consider that the clusters are statistically "spherical" as far as the dissociation is concerned. The observation of a $n^{2 / 3}$ dependence for the dissociation cross section reflects the invariability of the cluster compactness as $n$ varies, just like the $A^{1 / 3}$ dependence of the nuclear radius reflects the invariability of the density of the nuclear matter. The value found for $K$ leads to a density of 0.052 atoms $/ \AA^{3}$ if one makes the hypothesis that the dissociation cross section is equal to the geometrical cross section of "spherical" clusters. This value is in good agreement with the previous estimate by Van Lumig and Reuss $/ 5 /\left(0.025 \mathrm{H}_{2} / \AA^{3}\right)$. It is clear that more experimental work bearing particularly on the dissociation fragments would be needed to identify the various dissociation processes.

In a second group of experiments, we focused our attention on some consequences of the penetration of energetic clusters into thin carbon foils. These experiments generally involve a detailed analysis of emerging fragments and usually require a detailed analysis of the velocity distributions and of the charge state distribution of the emerging fragments. These distributions can be compared with measurements performed with atomic projectiles of the same velocity.

When an hydrogen ion cluster penetrates into a condensed medium most of its electrons are immediately stripped off and the binding between the atoms constituting the cluster is disrupted. The ionic fragments are repelled from each others by a screened Coulomb interaction while they fly through the solid. When they emerge from the exit surface of the foil some of these fragments bind electrons that may originate from the incident projectile or that have been picked up from the target. The first process is dominant in the case of very small dwell times in the foil and is directly related to the transmission probability of the projectile electrons $/ 6 /$. In the "pick-up" regime corresponding to thicker foils, the close proximity between the fragments at the exit surface tends to decrease their average final charge state /7-9/. In the particular case of hydrogen molecules, these effects are known to increase the neutralization probability of atomic fragments.

We have measured the angular distribution and the yield of the hydrogen fragments emerging from a self-supporting $2.1 \mu \mathrm{g} \cdot \mathrm{cm}^{-2}$ carbon foil bombarded with $\mathrm{H}_{\mathrm{n}}^{+}$projectiles at velocities ranging from 1.2 to $2.2 \mathrm{v}_{0} / 10,11 /$. In this velocity range the emergent fragments are mainly $\mathrm{H}^{\circ}$ and $\mathrm{H}^{+}$. We note that the target is thick enough to consider that all the emergent $H^{\circ}$ result from a capture of a target electron.

We show first (figure 2) the FWHM, $\theta_{0}^{n}$, of the angular distributions of neutral atoms for $n$ values up to 21 and for velocities between 30 and $120 \mathrm{keV} / \mathrm{p}$, after traversal of the $2.1 \mu \mathrm{g} \cdot \mathrm{cm}^{-2}$ carbon foil. For each velocity $\theta_{0}^{n}$ is seen to increase first with $n$ and then to reach a saturation value for $n \geq 5$. The enhancement above the $\theta_{0}^{1}$ value is due to the (screened) repulsion between the fragments inside the carbon foil, since it is quite reasonable to assume that the interaction in the post foil region between a neutral with either protons or neutrals is negligible. The saturation effect above $n \sim 5$, observed also for thicker foils, means that the average kinetic energy gained by each fragment of a $\mathrm{H}_{n}^{+}$cluster is the same when $n \geq 5$. Moreover the $n$ dependence of $\theta_{0}^{n}$ is quite smooth and does not confirm the assumption deduced from gas dissociation studies $/ 4 /$ about the possibility that 15 and 19 could be magic numbers corresponding to denser clusters. However multiple scattering could wash out such small effects.

In figure 3, we show for various velocities the variation of the neutral fraction $\phi_{0}^{n}$ with $n$ for a $2.1 \mu \mathrm{g} \cdot \mathrm{cm}^{-2}$ foil. As for $\theta_{0}^{n}, \phi_{0}^{n}$ is seen, at a given velocity, to increase first with $n$ and then to reach a saturation value. The difference with the previous case lies in the $n$ value corresponding to the saturation, 7 in this case, a value that is also nearly independent of the foil thickness. This value can be interpreted by considering that the mutual positions of the individual protons inside the exploding 
cluster are randomized in the target, due to both repulsion and milltiple scattering effects. Then if the protons are randomly distributed inside a sphere, the number of closest neighbours of a proton located at the center is 12. The number of closest neighbours is much less for a proton located in the outer part of the sphere, and the average number of closest neighbours is estimated to be 6.5 , quite in agreement with our observation.

So, with these foil experiments, we show that the information. one can extract are of two kinds. The angular distribution of the emergent fragments depends on what happens in the first layers of the foil and could shed some light on the structure of the incident clusters when multiple scattering effects are not too large. On the contrary, the charge state distribution at emergence depends strongly on the respective distances between the fragments in the last layers of the foil and may allow to help understanding the fundamental processes in the interaction of ionic projectiles with solid targets. The atomic excitation of the fragments after the break-up of ion clusters in thin carbon foils are being measured by conventional beam foil spectroscopy technique and will give new information on collective effects on atomic collisions in solids.

However, some theoretical and experimental difficulties are due to the too low velocity of our cluster ion beam. We know for example that multiple scattering effects severely limit the accuracy of the informations one can extract from the data, namely those concerning the cluster ion structure. The available ranges of cluster ion masses and energies are rather limited with the existing electrostatic accelerator. It has therefore been proposed to accelerate cluster ions by means of a Radio Frequency Quadrupole (RFQ) accelerator [H.O. Moser and A. Schempp, contributed paper to this Workshop]. The beam of $500 \mathrm{keV} \mathrm{H}{ }_{n}^{+}$cluster ions $(n \leqslant 50)$ produced in the Lyon accelerator will be injected into a RFQ post-accelerator designed to give a final energy of up to $5 \mathrm{MeV}$.

Finally, we would review quickly what could be done with faster $\mathrm{H}_{\mathrm{n}}^{+}$ions. We select only four topics : -- Cluster structure - When very thin foils and swifter ionic clusters are to be used, electron capture by protons at emergence will be negligible, the Coulomb repulsion between the protons will occur mainly in vacuum downstream the target, multiple scattering inside in the foil will be reduced and the joint angle-energy distributions of the break-up products will give a direct information on the structure of the incident cluster ion. Ion cluster dissociation induced by a transverse electron beam and by a gas jet will also be used for structure studies.

-- Cluster stopping power - One can give an interpretation of the energy loss of an ionic cluster in matter by dividing the electronic energy loss in two parts : the energy loss due to close collisions with individual target electrons - this part involves too small impact parameters to be concerned by any proximity effects - and the energy loss due to distant excitation of the target electrons in collective modes by the swarm of protons. This last process involves distances larger than the mean distance between protons in the swarm so that proximity effect can be manifested. This interpretation allows a quick estimation of the stopping power for an ion cluster but the theoretical limits between close and distant collisions are not accurate enough to allow a full quantitative description of the stopping power to be observed.

Another point of view is the following one. In the polarization wake concept the electronic stopping power of a medium for a fast ion $\left(v \geq v_{0}\right)$ is related to the derivative of the wake potential at the ion site. Then the energy loss of a ionic cluster should be determined by the interference of the individual wakes of each fragment in the cluster. This concept was first developed by Brandt and Ritchie /12/ 
in terms of a "vicinage function" calculated from interference effects between plasmon wakes. Due to the close proximity of the fragments in cluster beam-foil experiments with fast projectiles the interference should be observed. Indeed an enhancement has already been observed in various experiments $/ 13 /$ with molecular ions like $\mathrm{H}_{2}^{+}$or $\mathrm{H}_{3}^{+}$. It would be interesting to extend this study to larger systems such as ionic clusters.

-- Electron Spectroscopy - Although the production of electrons by fast ion impact is of principal interest in many branches of physics, it is only recently that electron spectroscopy has received a great deal of attention. In particular, the so-called cusp electrons which are emitted with nearly zero velocity relative to the projectile, were actively investigated both experimentally and theoretically /14\%. It would be interesting to compare the results obtained with heavy ions and with $\mathrm{H}_{n}^{+}$clusters of the same velocity.

- Channeling of fast ionic clusters will considerably modify the dynamics of the explosion inside the foil by breaking down its cylindrical symmetry. Planar channeling potential will prevent the repulsion along the transverse direction of protons channeled between the same atomic planes. Then channeling experiments should provide a unique way of sampling the incident projectile into slices of known transverse width (the planar spacing $\mathrm{dp}$ ), which should give informations on the structure and the vibration motion of the incident clusters.

New studies are now in progress and a new generation of experiments will be possible with the expected higher energy clusters, concerning the knowledge of the ionic clusters themselves as well as the investigation of collective effects in the particle-matter interaction.

11/ Henkes, W. Z. Naturforschq. 16a (1961) 842

12/ Yamaguchi, Y., Gaw, J.F., Regmington, R.B. and Schaefer, H.F. J. Chem. Phys. $\underline{86}$ (1987) 9

131 Chanut, Y., Martin, J., Salin, R., Moser, H.O. Surface Sci. 106 (1981) 563

14/ Chevallier, M., Clouvas, A., Frishkorn, H.J., Gaillard, M.J., Poizat, J.C. and Remillieux, J. Z. Phys. D-2 (1986) 87

15/ Van Lumig, A. and Reuss, J. Int. J. Mass. Spectrom. Ion Phys. 25 (1977) 137

16/ Gaillard, M.J., Poizat, J.C., Ratkowski, A., Remillieux, J. and Auzas, M. Phys. Rev. A-16 (1977) 2323

/7/ Maor, D., Cooney, P.J., Faibis, A., Kanter, E.P., Koenig, W., Zabransky, B.J. Phys. Rev. A-32 (1985) 105

/8/ Gaillard, M.J., Poizat, J.C., Ratkowski A. and Remillieux, J. Nucl. Instr. Meth. 132 (1976) 69

19/ de Castro Faria, N.V., Freire, F.L., Montenegro, E.C. and de Pinho, A.G. J. of Phys. B-19 (1986) 1781

/10/ Mazuy, B., Belkacem, A., Chevallier, M., Gaillard, M.J., Poizat, J.C. and Remillieux, J. Nucl. Instr. Meth. in Phys. Res. B-28 (1987) 497

/11/ Mazuy, B., Belkacem, A., Chevallier, M., Gaillard, M.J., Poizat, J.C. and Remillieux, J. Nucl. Instr. Meth. in Phys. Res. B (to be published)

/12/ Brandt, W. and Ritchie, R.H. Nucl. Instr. Meth. 132 (1976) 43

113/ See for example : Brandt, W., Ratkowski, A. and Ritchie, R.H. Phys. Rev. Lett. 33 (1974) 1329 ; Tape, J.W., Gibson, W.M., Remillieux, J., Laubert, R. and Wegner, H., Nucl. Instr. and Meth. 132 (1976) 75

/14/ Meckbach, W., Chiu, K.C.R., Brongersma, H.H. and McGowan, J.W. J. of Phys. B-10 (1977) 3255 